\title{
Identification and functional analysis of a novel splice variant of AC3-33 in breast cancer
}

\author{
LU YUAN ${ }^{1}$, FEN HU ${ }^{2}$, YUNFENG ZHANG ${ }^{3}$, LIJUN MENG ${ }^{4}$, \\ TIANYANG $\mathrm{AN}^{5}$, YAJING $\mathrm{CHEN}^{6}$ and XIUJUN ZHANG ${ }^{7}$ \\ ${ }^{1}$ College of Public Health; ${ }^{2}$ College of Life Sciences, North China University of Science and Technology, \\ Tangshan, Hebei 063000; ${ }^{3}$ Department of Life Sciences, Tangshan Normal University; ${ }^{4}$ Department of \\ Environmental and Chemical Engineering, Tangshan College; Colleges of ${ }^{5} \mathrm{Jitang},{ }^{6} \mathrm{Pharmacy}$ and \\ ${ }^{7}$ Psychology, North China University of Science and Technology, Tangshan, Hebei 063000, P.R. China
}

Received September 29, 2018; Accepted September 10, 2019

DOI: $10.3892 /$ etm.2019.8212

\begin{abstract}
Alternative RNA splicing plays a key role in regulating gene function and influencing protein expression diversity. In the present study, an AC-33 transcript variant (NCBI Reference Sequence: NM_001308229.1), splice variant (sv)AC3-33, was successfully cloned from the MCF-7 breast cancer cell line by reverse transcription PCR using primers based on expressed sequence tags. The aim of the present study was to investigate the structure and function of svAC3-33. svAC3-33 has an open reading frame of 1,825 base pairs, lacks AC3-33 exon 2 and is encoded by 294 amino acids. svAC3-33 is localized within the cytoplasm. The Cell Counting Kit- 8 and EdU detection of cell proliferation assays showed that svAC3-33 inhibited MCF-7 cell proliferation. Similarly, svAC3-33 knockdown by RNA interference was shown to have the opposite effect by repressing the cell cycle progression of breast cancer cells. Furthermore, the data indicated that svAC3-33 may upregulate the expression of p21. The present study provides evidence that the increased expression of svAC3-33 may inhibit the activity of the transcription factor AP-1. The luciferase reporter gene assay detected a downregulation of the expression of c-Jun, but not c-Fos, which in turn affected cell proliferation. In conclusion, these results indicated a function for svAC3-33 in inhibiting the cell
\end{abstract}

Correspondence to: Dr Xiujun Zhang, College of Psychology, North China University of Science and Technology, 57 Jianshenan Road, Tangshan, Hebei 063000, P.R. China

E-mail: xiujunzhang66@126.com

Dr Fen Hu, College of Life Sciences, North China University of Science and Technology, 57 Jianshenan Road, Tangshan, Hebei 063000, P.R. China

E-mail: ihufen@126.com

Key words: splice variant chromosome 3 open reading frame 33, MCF-7 cells, cell proliferation, 5-ethynyl-2'-deoxyuridine assay, cyclin dependent kinase inhibitor 1, c-Jun proliferation of MCF-7 cells by regulating the AP-1 signaling pathway.

\section{Introduction}

Based on high-throughput screening techniques, a novel human gene, chromosome 3 open reading frame 33 (AC3-33), capable of inhibiting phorbol 12-myristate 13 -acetate and AP-1 activation, has been identified from 650 known human genes (1). Previously, it was demonstrated that the AC3-33 gene is widely expressed in the adrenal gland and cervix (2). Further studies on AC3-33 indicate that it is a secretory protein that inhibits ETS transcription factor ELK1 transcriptional activity through the ERK1/2 pathway $(2,3)$. Alternative RNA splicing not only increases the diversity of mRNA expression, but also plays an important role in the regulation of gene function $(4,5)$. Wang et al $(6)$ found that a single-block intronic expressed sequence tag (EST) containing a polyadenylation site could form a 3 'exon site, thus forming transcript variants. Although one AC3-33 transcript variant has previously been reported (1), previous data suggested that other AC3-33 isoforms may exist.

Breast cancer is the most common cancer in women worldwide, and its incidence is increasing, making breast cancer a major public health problem (7). Numerous signaling pathways can modulate the development of breast cancer cells, which can affect the cell cycle as well as processes involving the activation and inhibition of specific genes. The activation and inhibition of the transcription factor AP-1 can greatly affect the growth and reproduction of cancer cells, regulating the development of many deadly cancer types $(7,8)$. AP-1 is mainly composed of the c-Jun, c-Fos, MAF and activating transcription factor protein families. In human cells, AP-1 comprises c-Jun and c-Fos, which can activate and affect numerous signaling pathways, in addition to regulating cell growth and reproduction (9-15). Previous studies have demonstrated that infection, growth factors and cancer cells affect the expression of AP-1-related signaling pathway, leading to the division, differentiation and apoptosis of cancer cells (16-19).

In the present study, a second $\mathrm{AC} 3-33$ transcript variant was successfully cloned, splice variant (sv)AC3-33. The data 
also characterized svAC3-33 and demonstrated the subcellular localization of the encoded protein. Furthermore, the effect of raised svAC3-33 expression on cell proliferation was demonstrated. Our present evidence shows that svAC3-33 may inhibit MCF-7 cell progression by downregulating c-Jun, which is an important member of the AP-1 signaling pathway.

\section{Materials and methods}

PCR identification. Human breast cancer cell line MCF-7 and human cervical carcinoma cell line HeLa were purchased from the American Type Culture Collection and cultured in DMEM (Gibco, Thermo Fisher Scientific, Inc.) supplied with $10 \%$ FBS and penicillin/streptomycin (Gibco, Thermo Fisher Scientific, Inc.) at $37^{\circ} \mathrm{C}$ in a humidified $5 \% \mathrm{CO}_{2}$. TRIzol reagent (Invitrogen; Thermo Fisher Scientific, Inc.) was used to extract the total RNA from MCF-7 and HeLa cells. M-MLV reverse transcriptase (Promega Corporation) was used for RT-qPCR, and the first strand cDNA was synthesized. For each sample, cDNA synthesis was performed using $0.25 \mathrm{mg}$ of total RNA and PrimeScript RT Master Mix Perfect RT (Takara Bio, Inc.). The cDNA of MCF-7 was used to amplify sv-AC3-33, and the cDNA of HeLa was used to amplify AC3-33. svAC3-33 and AC3-33 were amplified by PCR using the following primers: Forward 5'-GAGGAGCTCAGGGCC GC-3' and reverse 5'-TAAAGCATAAAGAATTCCTTTA-3'. PCR amplification was conducted using a Sangon Biotech PCR kit (cat. no. B639297; Sangon Biotech Co., Ltd.). According to the manufacturer's instructions, DNA template $0.5 \mu 1$, primer F $1 \mu 1$, primer R $1 \mu 1$, Taq PCR Master Mix $12.5 \mu \mathrm{l}$ and $\mathrm{ddH}_{2} \mathrm{O}$ up to $25 \mu \mathrm{l}$. Briefly, after an initial denaturation step at $95^{\circ} \mathrm{C}$ for $5 \mathrm{~min}$, amplifications were carried out with 31 cycles, consisting of a melting step at $95^{\circ} \mathrm{C}$ for $30 \mathrm{sec}$, an annealing step at $55^{\circ} \mathrm{C}$ for $30 \mathrm{sec}$, and an extension step at $72^{\circ} \mathrm{C}$ for $2 \mathrm{~min}$, followed by an extra extension step at $72^{\circ} \mathrm{C}$ for $5 \mathrm{~min}$. The PCR product was subjected to electrophoresis on a $1 \%$ agarose gel and sequenced by Sangon Biotech Co., Ltd.

Plasmid construction. A possible novel AC3-33 isoform was identified in the University of California Santa Cruz Genome Browser sequence database (http://genome.ucsc. edu/cgi-bin/hgGateway). svAC3-33 and full-length (or wild-type) AC3-33 are two alternatively spliced transcripts of the AC3-33 gene containing different open reading frames (ORFs). The full-length fragment of svAC3-33 cDNA was obtained by RT-PCR from MCF-7 cells using the following primers: Forward 5'-TATAAGCTTATGGCGGGGCAGCCC GCG-3' and reverse 5'-TATGGATCCCACCCTTTTCTACGA AAGTTT-3'. BamHI and HindIII recognition sites were added to the primers. The purified PCR product was recombined with p-enhanced green fluorescent protein (EGFP)-C3 vector (BD Biosciences) to form a recombinant expression vector. To construct pEGFP-C3-svAC3-33, the corresponding primers were used for fusion with the N-terminal GFP tag in the PEGFP-C3 vector with BamHI and HindIII. All clones were confirmed by sequencing using an ABI Prism 3100 Genetic Analyzer (Applied Biosystems; Thermo Fisher Scientific, Inc.). After confirmation, all plasmids were extracted and purified for transfection using EndoFree Plasmid Maxi Kit
(Qiagen, USA). Signal IP 4.0 Server (http://www.cbs.dtu. $\mathrm{dk} /$ services/SignalP) was used to analyze the signal peptide of the svAC3-33 protein; TMHMM server v 2.0 (http://www. cbs.dtu.dk/services/TMHMM-2.0/) was used to predict the transmembrane location of proteins.

Preparation of the small interfering (si)RNA. The siRNA sequence for the svAC3-33 gene was designed and synthesized. This siRNA sequence was located in the ORF of the svAC3-33 gene, and the target sequence of the siRNA was 5'-GGACGAUUACGCCGAAUAA-3'. A nonsense sequence siRNA was used as a control, named si-NC. Sense and antisense strands were designed and synthesized by Beijing Genomics Institute. According to the manufacturer's instructions (pSilencer 4.1-CMV neo siRNA Expression Vector kit), strands were annealed to the corresponding sites of pSilencer 4.1-CMVneo (Thermo Fisher Scientific, Inc.; www.ambion. com/techlib/msds) HindIII and BamHI, thus constructing a silent plasmid, named si-svAC3-33.

Cell culture and transient transfection. MCF-7 cells were supplemented with 10\% FBS (Gibco; Thermo Fisher Scientific, Inc.) and $1 \%$ penicillin-streptomycin in DMEM (Gibco; Thermo Fisher Scientific, Inc.), and maintained at $37^{\circ} \mathrm{C}$ with $5 \% \mathrm{CO}_{2}$. Then $2 \times 10^{4}, 5 \times 10^{4}$ and $1.2 \times 10^{4} \mathrm{MCF}-7$ cells were seeded into six-well, 24-well or 96-well plates, respectively, and transiently transfected with pEGFP-C3, pEGFP-C3-svAC3-33, si-NC or si-svAC3-33 plasmids using and Lipofectamine ${ }^{\circledR} 2000$ (all, Thermo Fisher Scientific, Inc.) was used according to the manufacturer's protocol.

Subcellular localization.pEGFP-C3 and pEGFP-C3-svAC3-33 plasmids were transiently transfected into MCF-7 cells in a 6-well plate, and then each well of the cells were washed twice with PBS. After transfection MCF-7 cells were incubated at $37^{\circ} \mathrm{C}$ for $24 \mathrm{~h}$, then $4 \%$ paraformaldehyde was used to fix MCF-7 cells for $30 \mathrm{~min}$ at room temperature, and DAPI was added to stain the nuclei for $2 \mathrm{~min}$ at room temperature. After washing twice with PBS, images of MCF-7 cells were captured (magnification x400; IX71 inverted fluorescence microscope; Olympus Corporation) and analyzed using ImageJ (version 1.40; National Institutes of Health).

Cell Counting Kit-8 (CCK-8) assay. pEGFP-C3, pEGFP-C3-svAC3-33, si-NC or si-svAC3-33 plasmids were transiently transfected into $\mathrm{MCF}-7$ cells as previously described. After transfection MCF-7 cells were incubated at $37^{\circ} \mathrm{C}$ for $24 \mathrm{~h}, 2,000$ cells were seeded into 96-well plate. CCK-8 solution (10 $\mu \mathrm{l}$; Beijing Zoman Biotechnology Co.,Ltd.) was added to each well, and the cells were incubated at $37^{\circ} \mathrm{C}$, $5 \% \mathrm{CO}_{2}$ for $2 \mathrm{~h}$ according to the manufacturers protocol. The absorbance at $450 \mathrm{~nm}$ was measured daily with a microplate reader for 5 days.

EdU cell proliferation assay. MCF-7 cells were transiently transfected with pEGFP-C3, pEGFP-C3-svAC3-33, si-NC or si-svAC3-33 plasmids in 96-well plates at 50-60\% confluence of $5 \times 10^{4}$. The steps of cell incubation and staining were based on the manufacturer's instructions. MCF-7 cells were fixed using $4 \%$ paraformaldehyde for $30 \mathrm{~min}$ at room temperature, 
washed with $0.5 \%$ Triton $\mathrm{X}-100$ three times, and images were captured using an IX71 inverted fluorescence microscope (magnification, x200, IX71 inverted fluorescence microscope; Olympus Corporation).

Dual-luciferase assay to measure AP-1 activity. MCF-7 cells were co-transfected with pEGFP-C3 or pEGFP-C3-svAC3-33; si-NC (nonsense siRNA) or si-svAC3-33 and pAP-1-Luc plasmid and pGL3-Basic plasmid which contain the Renilla luciferase gene to normalize the transfection efficiency (all, Promega Corporation). After transfection the MCF-7 cells were incubated for $24 \mathrm{~h}$ at $37^{\circ} \mathrm{C}$, and cells were lysed and assayed. Luciferase activities were tested using the Dual-Luciferase ${ }^{\circledR}$ Reporter Assay System (Promega Corporation) following the manufacturer's instructions and measured using a microplate luminometer reader (Tecan Group, Ltd.). Three independent repeat tests were performed.

Western blot analysis. MCF-7 cells were transfected with si-NC or si-svAC3-33, pEGFP-C3 or pEGFP-C3-svAC3-33, and $\mathrm{MCF}-7$ cells were incubated at $37^{\circ} \mathrm{C}$ for $48 \mathrm{~h}$, then total cell extracts and the western blotting was performed as previously described (20). The total protein concentration was determined using the BCA protein assay kit (Sangon Biotech Co., Ltd.). Specific antibodies for the following proteins were used: Anti-green fluorescent protein (1:5,000; cat. no. D110008; BBI Solutions), anti-c-Fos (1:500; cat. no. D120415; BBI Solutions), anti-c-Jun (1:500; cat. no. D155181; BBI Solutions) and anti- $\beta$-actin (1:50,000; cat. no. AC026; ABclonal Biotech Co., Ltd.). Bands were visualized using an ECL kit (Sangon Biotech Co., Ltd.).

$R N A$ extraction and reverse transcription-quantitative $P C R$ $(R T-q P C R)$. TRIzol ${ }^{\circledR}$ reagent (Invitrogen; Thermo Fisher Scientific, Inc.) was used to extract the total RNA from MCF-7 cells. M-MLV reverse transcriptase (Promega Corporation) was used for RT-PCR, and the first strand cDNA was synthesized (9). Primers were designed and synthesized by Beijing Genomics Institute, and the primers were as follows: p15, forward 5'-CTAGTGGAGAAGGTGCGACA-3' and reverse 5'-ACCAGCGTGTCCAGGAAG-3'; p21, forward 5'-CCC GTGAGCGATGGAACT-3' and reverse 5'-AGGCACAAG GGTACAAGACA-3'; p27 forward 5'-TAGAGGGCAAGT ACGACGAGTGG-3' and reverse 5'-CAGGTCGCTTCCTTA TTCC-3'; p53 forward 5'-CCTCCTCAGCATCTTATCCG-3' and reverse 5'-CACAAACACGCACCTCAAA-3' GAPDH, forward 5'-AAAGGGTCATCATCTCTG-3' and reverse 5'-GCTGTTGTCATACTTCTC-3'. qPCR was performed with the designed primers. The reaction was carried out using a 7900HT Fast Real-Time PCR System (Thermo Fisher Scientific, Inc.). GAPDH-80 was used as the internal control. Amplification was performed under the following conditions: $1 \mathrm{~min}$ at $95^{\circ} \mathrm{C} ; 5 \mathrm{sec}$ at $95^{\circ} \mathrm{C} ; 30 \mathrm{sec}$ at $60^{\circ} \mathrm{C}$ for 40 cycles; Melt Curve 65 to $95^{\circ} \mathrm{C}$. Gene expression levels were calculated as a ratio of the expression of GAPDH, and data were analyzed using the $2^{-\Delta \Delta \mathrm{Ct}}$ method (21).

Statistical analysis. All of the data were collected from three independent experiments. Statistical analyses were carried out using one-way ANOVAs followed by post hoc Tukey tests, or
Student's t-tests. $\mathrm{P}<0.05$ was considered to indicate a statistically significant difference.

\section{Results}

Cloning and characterization of the svAC3-33 transcript variant. The novel human gene AC3-33 is a secretory protein, and can suppress the activity of the transcription factor AP-1 (1). In the present study, specific primers were used based on an EST from AC3-33, to clone a novel splice variant of AC3-33 cDNA [the PCR product is $~ 1,825$ base pairs (Fig. 1A)] from MCF-7 cells, which was designated svAC3-33. Only svAC3-33 was amplified, and no expression of AC3-33 was noted (Fig. 1A). Therefore, the effects of this siRNA on cell proliferation were due to the silencing of the svAC3-33 transcript (Fig. 3A). Full-length AC3-33 and svAC3-33 transcripts are the alternatively spliced transcripts of the AC3-33 gene, which contains different ORFs (Fig. 1B). The putative svAC3-33 protein contains 294 amino acids (Fig. 1C) and has a predicted molecular mass of $29 \mathrm{kDa}$. Signal IP 4.0 Server analysis revealed that the svAC3-33 contained no signal peptides, whereas TMНМM server v. 2.0 analysis revealed that the svAC $3-33$ protein does not contain a transmembrane spanning region. The $\mathrm{C} 3$ orf 33 gene is located on human chromosome 3q25.31. According to the NCBI database (NM_173657.2 and NM_001308229.1 https://www.ncbi.nlm.nih.gov/nuccore/NM_001308229.2/), svAC3-33 comprises five exons and four introns. svAC3-33 is very similar to AC3-33, except that it lacks exon 2 entirely and has a pre-translation initiation site, resulting in the coding sequence starting earlier than AC3-33 (Fig. 1C). There are currently no studies indicating the function of svAC 3-33'.

Subcellular localization of svAC3-33. A pEGFP-C3-svAC3-33 plasmid was constructed and transiently transfected it into MCF-7 cells. svAC3-33 was mainly located in the cytoplasm (Fig. 2A) and the size of the pEGFP-C3-svAC3-33 encoded protein was around $56 \mathrm{k}$ Da (Fig. 2B).

svAC3-33 expression inhibits cell proliferation. Western blotting indicated that svAC3-33 was expressed in MCF-7 cells following transfection (Fig. 2B). MCF-7 cells were co-transfected with pEGFP-C3-svAC3-33 and si-NC, pEGFP-C3-svAC3-33 and si-svAC3-33 for $48 \mathrm{~h}$. pEGFP-C3-svAC3-33 expression was qualitatively observed to be inhibited by si-svAC3-33 at the protein level (Fig. 3A). Counting of cell numbers with CCK- 8 indicated that the expression of svAC3-33 inhibited the proliferation of MCF-7 cells compared with those of the negative control group. Similarly, inhibiting endogenous svAC3-33 expression with siRNA may reduce the proliferation of MCF-7 cells (Fig. 3B). EdU+ cell numbers in svAC3-33-expressing cells decreased significantly after 2 days, compared with those of cells transfected with the empty vector (Fig. 3C). The EdU cell proliferation assay revealed a marked decrease in the number of MCF-7 cells in the S phase following transfection with pEGFP-C3-svAC3-33 (Fig. 3C). The EdU assay indicated that the percentage of cells in the $\mathrm{S}$ phase decreased from $30.27 \%$ in the transfection with control cells to $23.96 \%$ 


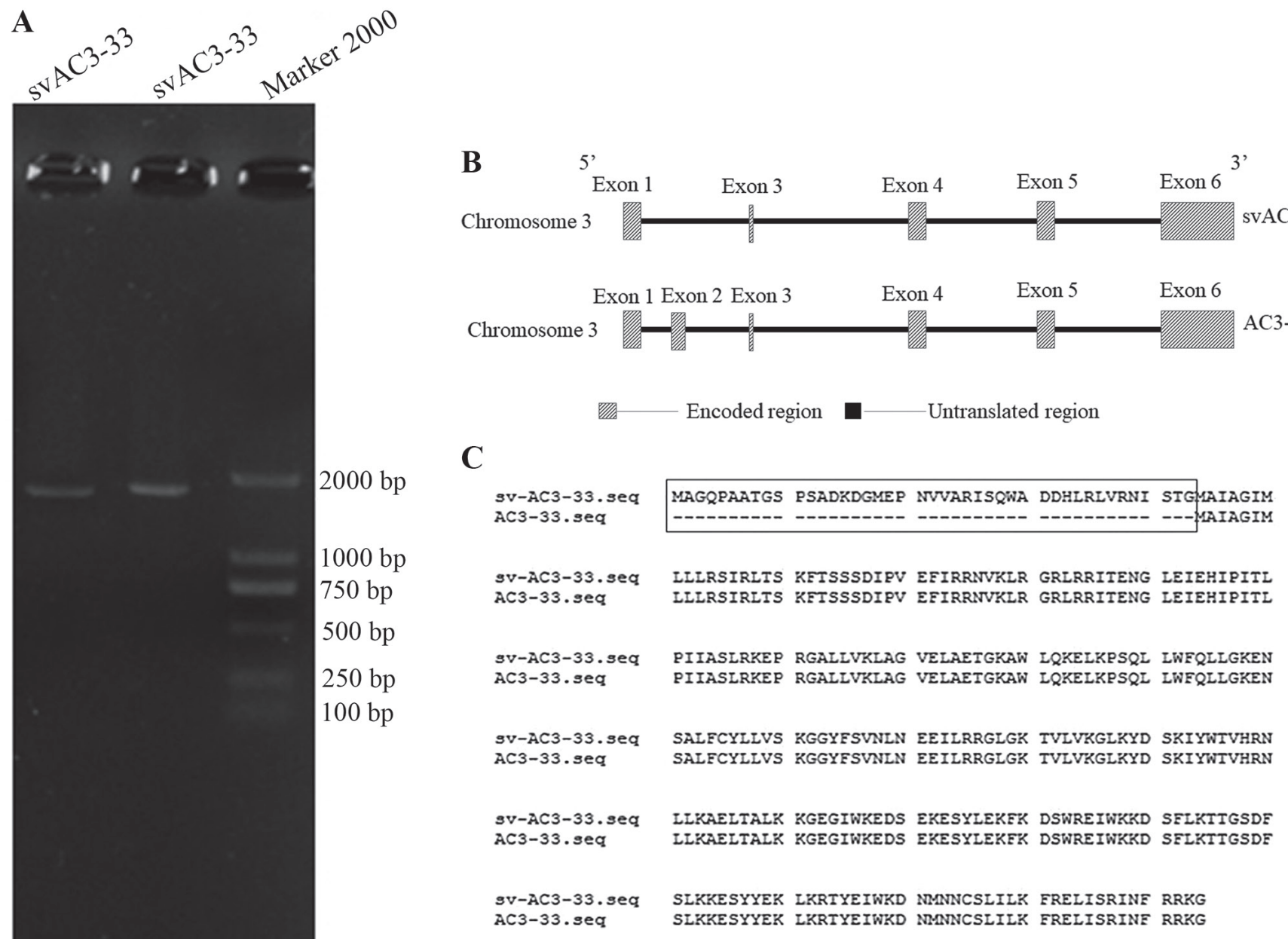

in pEGFP-C3-svAC3-33 cells at $48 \mathrm{~h}$ following transfection. treating pEGFP-C3-svAC3-33+ cells with si-svAC3-33, compared with si-NC treated cells, increased the percentage of cells in the $\mathrm{S}$ phase from 25.37 to $40.21 \%$ at $48 \mathrm{~h}$ (Fig. 3D). Taken together, these data suggested that svAC3-33 may inhibit the cell cycle progression of MCF-7 cells from the $\mathrm{S}$ phase.

To investigate the relationship between svAC3-33 and cell proliferation, the impact of svAC3-33 on the mRNA expression of p15, p21, p27, p53 and proliferation-related genes was examined. It was found that svAC3-33 only regulates p21 expression (Fig. 3E-F). Compared with the pEGFP-C3 transfected negative control group, the expression of $\mathrm{p} 21$ mRNA in the svAC3-33+ group was significantly higher than that in the control group. After silencing svAC3-33 with si-svAC3-33, compared with the si-NC negative control group, the mRNA expression levels of p21 were downregulated by $60 \%$ at $48 \mathrm{~h}$ (Fig. 3G).
Effects of svAC3-33 on AP-1 signaling. The role of the AP-1 signaling pathways in the antiproliferative function of svAC3-33 was subsequently examined. The dual-luciferase reporter assay was used to investigate the function of svAC3-33. Increased expression of svAC3-33 inhibited the activity of AP-1 compared with the transfection with empty vector controls in MCF-7 cells. Compared with the transfection with the si-NC-treated control, svAC3-33 knockdown by RNA interference increased the activity of AP-1 (Fig. 4A). c-Jun and c-Fos are the two important components of the transcription factor AP-1. Western blot analysis was used to determine which components of the specific transcription factor mediated the process wherein svAC3-33 suppressed the AP-1 activity (Fig. 4B). The expression levels of c-Jun protein were reduced by the increased expression of svAC3-33 (Fig. 4C), whereas there was no significantly change in the protein expression levels of c-Fos (Fig. 4D). These data demonstrate that svAC3-33 knockdown by RNA interference caused the upregulation of c-Jun, but not c-Fos. 
A
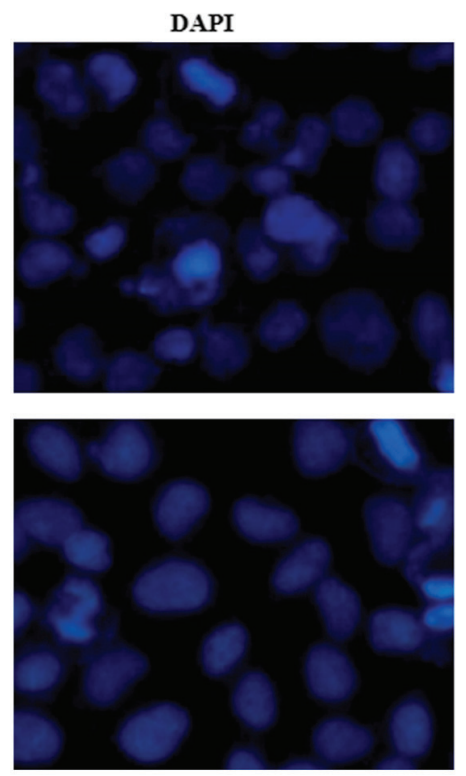
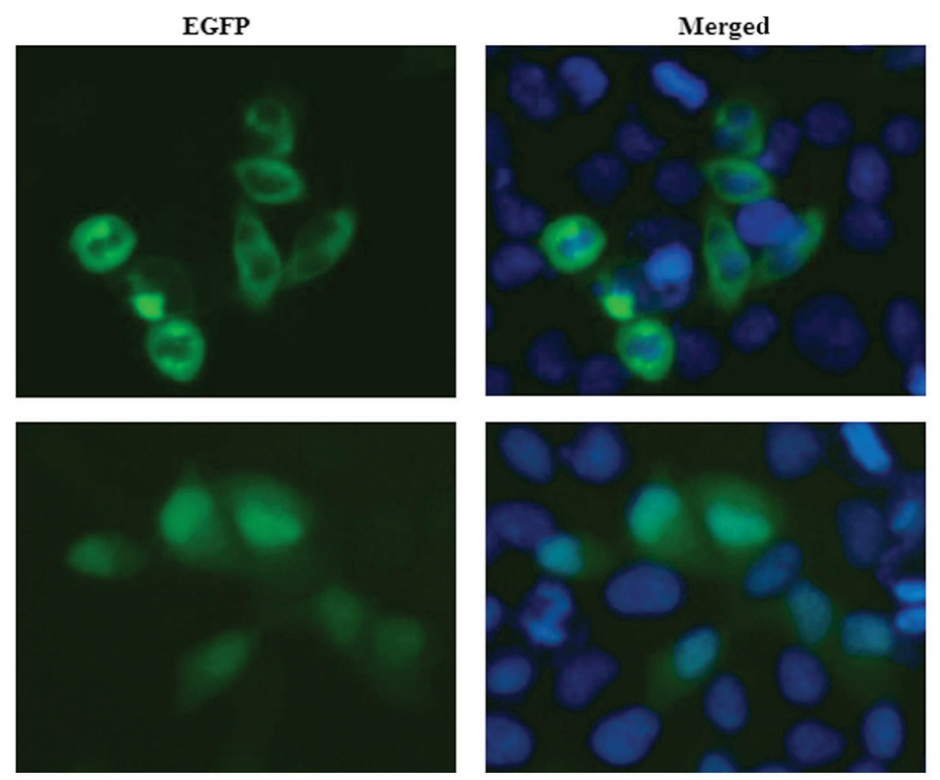

pEGFP-C3-svAC3-33

pEGFP-C3

\section{B}

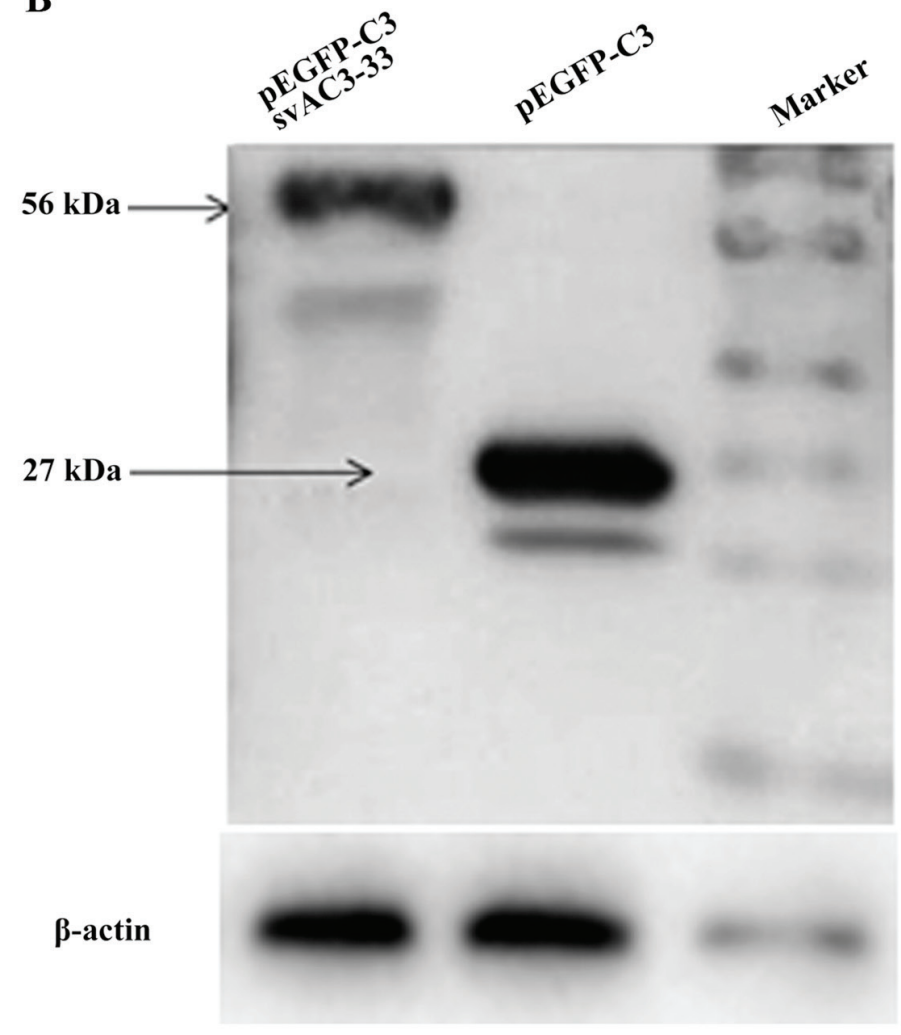

$72 \mathrm{kDa}$

$55 \mathrm{kDa}$

$43 \mathrm{kDa}$

34 kDa

$26 \mathrm{kDa}$

$17 \mathrm{kDa}$

$43 \mathrm{kDa}$

Figure 2. (A) Subcellular localization of svAC3-33. MCF-7 cells were transiently transfected with pEGFP-C3-svAC3-33 and pEGFP-C3 plasmid, and DAPI was used to stain nuclei. GFP and DAPI were visualized by fluorescence microscopy (magnification x400). (B) pEGFP-C3, pEGFP-C3-svAC3-33 plasmids were transiently transfected into MCF-7 cells. Western blot analysis was performed to analyze the EGFP conjugated protein expression levels. EGFP, enhanced green fluorescent protein; sv, splice variant.

\section{Discussion}

Breast cancer is one of the deadliest cancer types worldwide. Breast cancer results from cell cycle disorganization that leads to uncontrolled cellular pro liferation (7). Several studies have reported that in breast cancer, the expression levels of various growth factors are altered, which contributes to tumor progression and proliferation. Studies have shown that AP-1 plays a critical role in mediating the proliferation of breast cancer cells. It is estimated that $40-60 \%$ of human genes have alternatives vs. Alternative RNA splicing is able to modulate the functions of genes by increasing the number of transcripts, and therefore the number of proteins, that come from a single gene (22-25). The process of alternative splicing is regulated by a number of splicing factors, whose expression and activity are tightly regulated during development, cell cycle progression and cell differentiation. Previous studies have demonstrated that intron removal from 

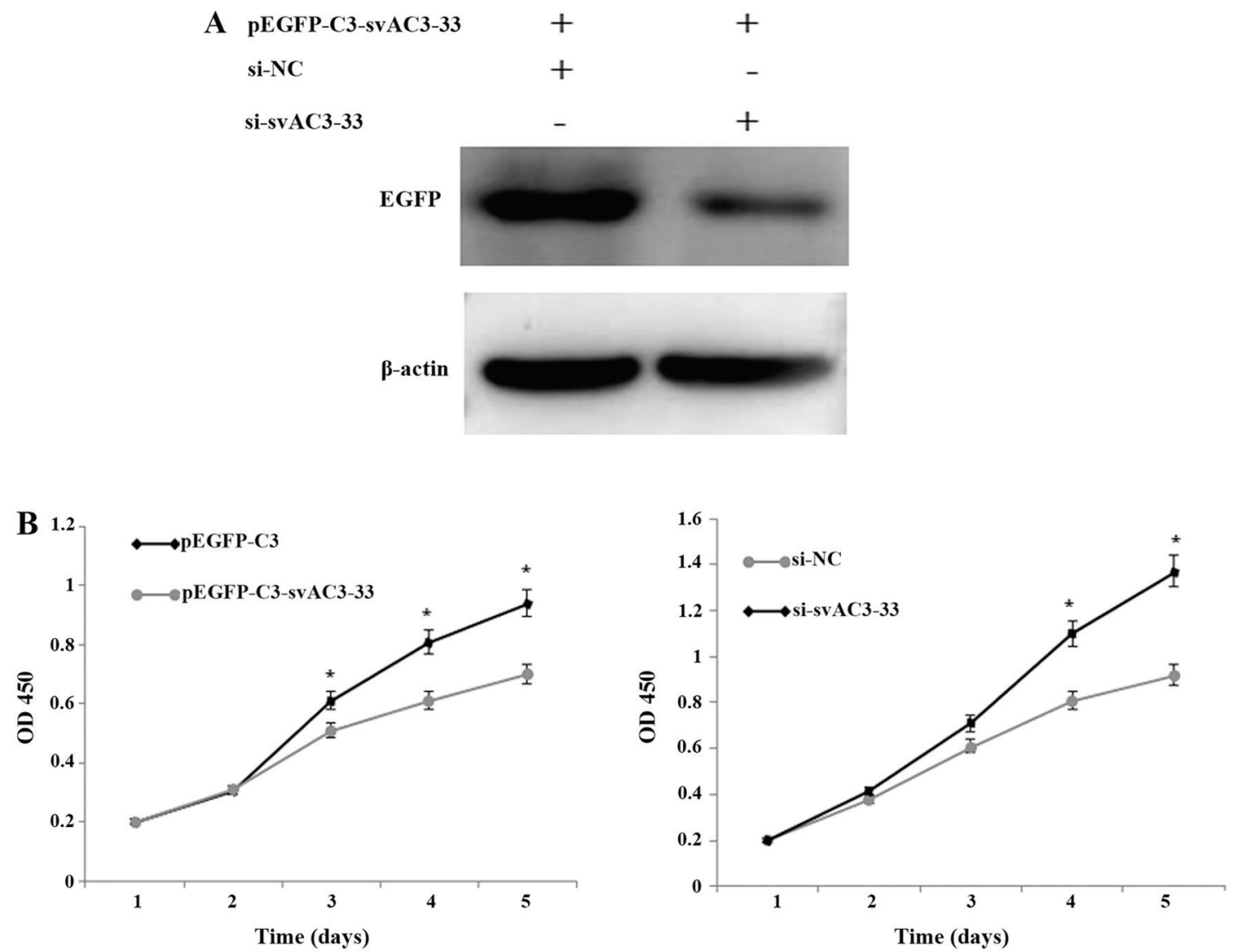

C
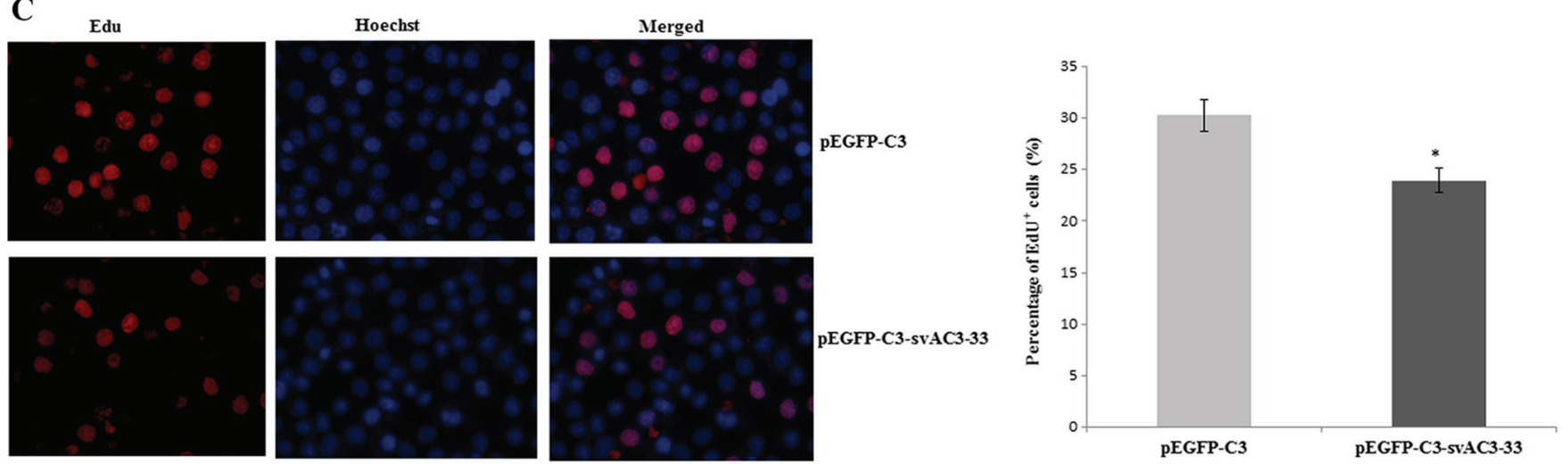

D
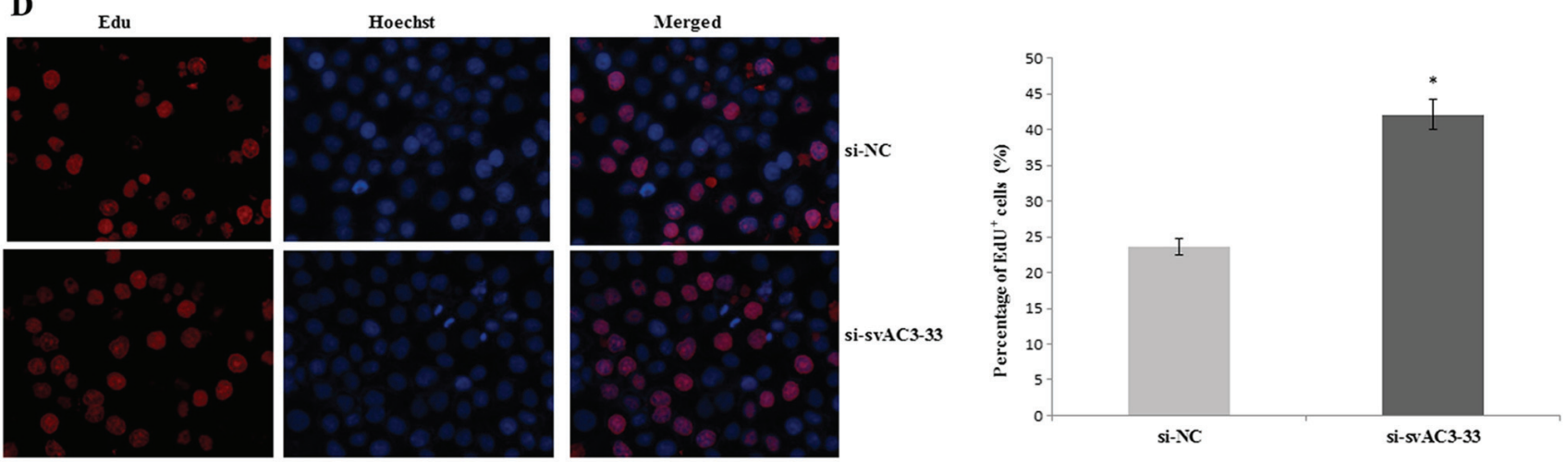

Figure 3. (A) MCF-7 cells were co-transfected with pEGFP-C3-svAC3-33 and si-NC, pEGFP-C3-svAC3-33, and si-svAC3-33 for 48 h. The expression levels of EGFP were measured by western blotting. $\beta$-Actin was used as a loading control. (B) MCF-7 cells were transfected with pEGFP-C3, pEGFP-C3-svAC3-33, si-NC, or si-svAC3-33, then assayed with a CCK-8 kit. Cells were collected and analyzed every $24 \mathrm{~h}$ and the absorbance was measured at $450 \mathrm{~nm}$. MCF-7 cells were transiently transfected with (C) pEGFP-C3 or pEGFP-C3-svAC3-33 as well as (D) si-NC or si-svAC3-33. Cell proliferation was measured by calculating the $\%$ of EdU+ cells $48 \mathrm{~h}$ after transfection (magnification, $\mathrm{x} 200$ ). Data were collected from three independent experiments. "P $<0.05$ vs. respective control. EGFP, enhanced green fluorescent protein; siRNA, small interfering RNA; si-NC, nonsense siRNA; si-svAC3-33, siRNA targeting svAC3-33; sv, splice variant. 

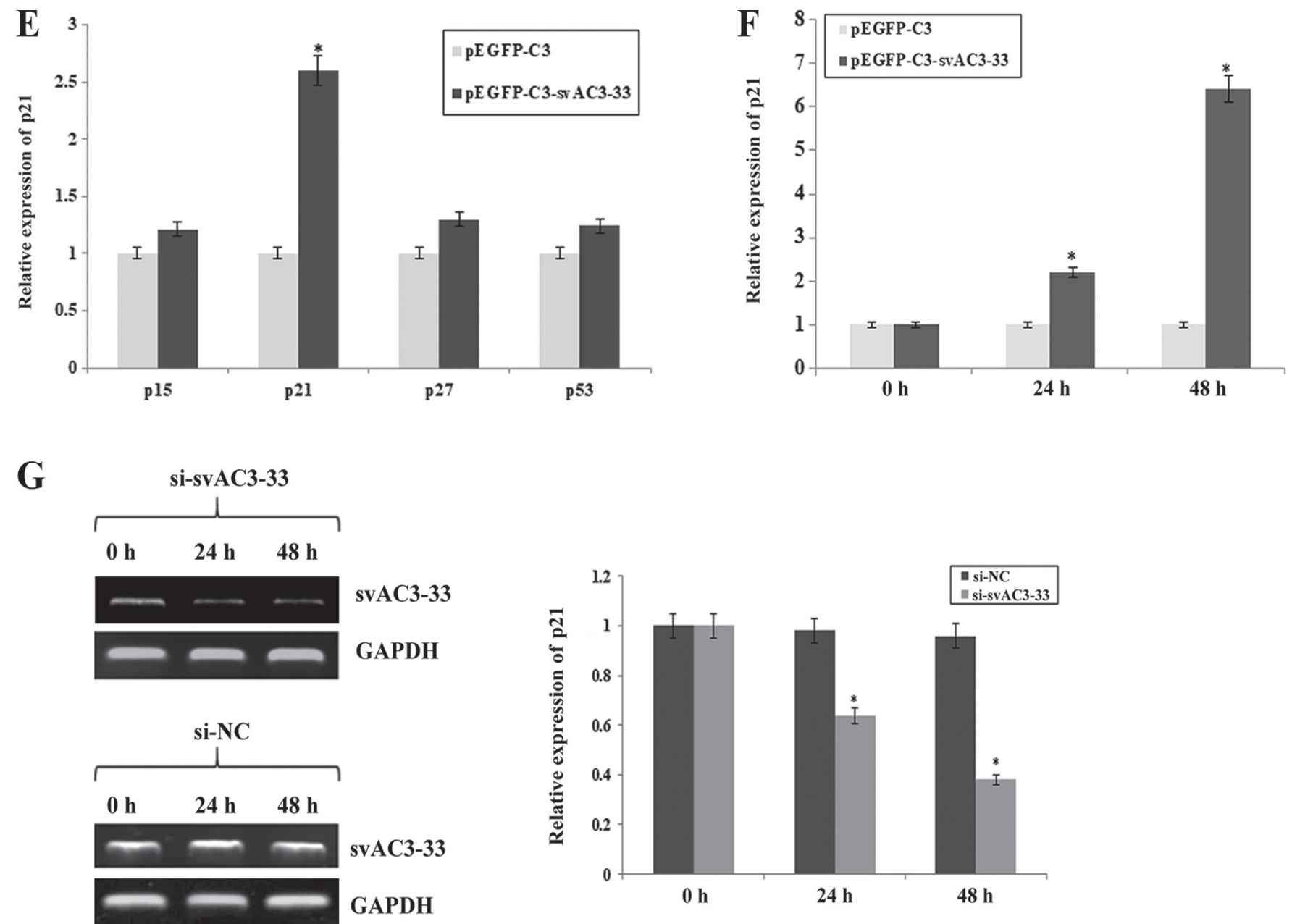

Figure 3. Continued. (E) MCF-7 cells were transfected with pEGFP-C3 or pEGFP-C3-svAC3-33 for $24 \mathrm{~h}$ and the expression of p15, p21, p27 and p53 was evaluated by RT-qPCR. However, no significant differences were found of p15, p27 and p53 compared with pEGFP-C3 group (P>0.05). (F) MCF-7 cells were transfected with pEGFP-C3 or pEGFP-C3-svAC3-33 for $24 \mathrm{~h}$. The expression of p21 at the mRNA level was measured at different time points $(0,24$, and $48 \mathrm{~h})$ after transfection, using RT-qPCR. GAPDH was used to normalize the p21 level. (G) si-svAC3-33 was transfected into MCF-7 cells. Control MCF-7 cells were treated with a scrambled siRNA. The efficiency of svAC3-33 mRNA knockdown was examined by RT-semiquantitative PCR. Compared with the si-NC control group, svAC3-33 was downregulated after transfection of si-svAC3-33 group. The inhibition of p21 mRNA level was determined at different time points $(0,24$ and $48 \mathrm{~h})$ after transfection, using RT-qPCR. GAPDH was used to normalize the p21 level, and then values were further normalized against the corresponding $0 \mathrm{~h}$ levels. Data were collected from three independent experiments. " $\mathrm{P}<0.05$ vs. respective control. EGFP, enhanced green fluorescent protein; RT, reverse transcription; q, quantitative; siRNA, small interfering RNA; si-NC, nonsense siRNA; si-svAC3-33, siRNA targeting svAC3-33; sv, splice variant.

a pre-mRNA by RNA splicing was initially thought to be controlled by intron splicing signals $(22,26)$, the regulation of alternative splicing is closely related to the function of cells. Alternative RNA splicing is mainly mediated and executed by exonic splicing enhancers as well as suppressors or silencers $(27,28)$.

p21 is an important member of the family of cyclin-dependent kinase inhibitors. It is closely related to tumor inhibition and can coordinate the relationship between the cell cycle, and DNA replication and repair, thus closely linking tumor suppression with the cell cycle. Under cellular stress conditions, p53 positively modulates p21. In turn, p21 exerts an inhibitory effect on cell-dependent kinases, thereby preventing cell cycle progression through the detection of DNA damage. It has been reported that the overexpression of estrogen receptor $\alpha$ in MCF-7 cells not only directly suppresses the activity of the p21 promoter, but also reduces the expression of p21 at both the mRNA and protein levels (29). It has been reported that the sustained arrest on the cell cycle caused by raised p21 expression could only be achieved when $\mathrm{p} 53$ was present in the cell and capable of transcriptionally activating the cyclin dependent kinase inhibitor 1A or p21 (30). Downregulating certain cyclins and upregulating of p21 and p27 at the transcript level may promote cell cycle arrest, and in turn promote growth inhibition (31).

Many studies have shown that $\mathrm{p} 21$ is a potent regulator of cell proliferation in different cell cultures. For example, in NIH3T3 cells, the hepatitis C virus (HCV) core protein inhibits the transcription of $\mathrm{p} 21$ by the transforming growth factor (TGF)- $\beta$ response element on the p21 promoter, indicating that the $\mathrm{HCV}$ core protein inhibits transcription by the inhibition of the TGF- $\beta$ pathway, thereby promoting cell cycle progression (32). c-Jun, a member of the transcription factor AP-1 family, is a key inducer of hepatocyte proliferation, which regulates liver regeneration by inhibiting p21 activity (33). These results are consistent with the present findings that svAC3-33 may contribute to the upregulation of $\mathrm{p} 21$ at the mRNA level in MCF-7 breast cancer cells. 

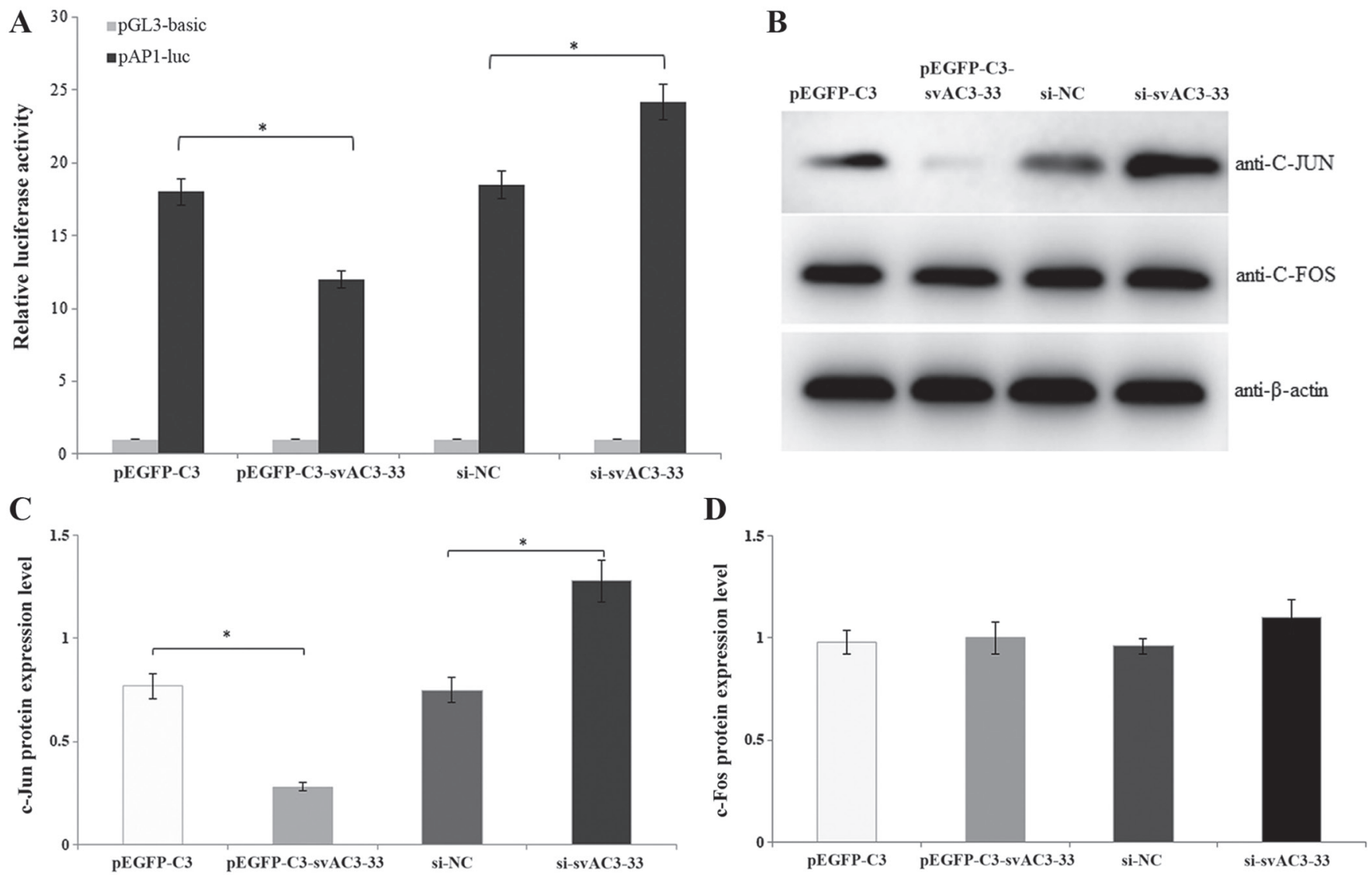

Figure 4. (A) pEGFP-C3, pEGFP-C3-svAC3-33, si-NC or si-svAC3-33 was transiently transfected into MCF-7 cells, and co-transfection with the firefly reporter plasmid and a Renilla luciferase vector for normalization. Cell lysates were tested for both firefly and Renilla luciferase activities with the dual luciferase assay. (B) MCF-7 cells were transfected with pEGFP-C3, pEGFP-C3-svAC3-33, si-NC and si-svAC3-33 for $48 \mathrm{~h}$. The c-Jun, and c-Fos protein expression levels were analyzed by western blotting. The protein expression levels of (C) c-Jun and (D) c-Fos were semi-quantified by western blotting. "P<0.05. EGFP, enhanced green fluorescent protein; luc, luciferase; siRNA, small interfering RNA; si-NC, nonsense siRNA; si-svAC3-33, siRNA targeting svAC3-33; sv, splice variant.

In this present study, a novel splice variant of AC3-33 was successfully cloned, designated svAC3-33. Notably, svAC3-33 and AC3-33 have different structures but have the same subcellular localization; they are both predominantly expressed in the cytoplasm (2). In the present study, the contribution of svAC3-33 to the regulation of MCF-7 cell proliferation was identified. The present study also examined the effect of svAC3-33 on the transcriptional activity of AP-1. It was demonstrated in this present study that increasing the expression of svAC3-33 caused the inhibition of AP-1. This also promoted the translocation of AP-1 into the nucleus and the binding of AP-1 with DNA in MCF-7 cells. A Previous study indicated that the transcriptional activity of AP-1 is mainly mediated by c-Jun, and c-Fos (34). Additionally, this present study identified that SvAC3-33 exerted its functional role through the c-Jun signaling pathway, but not through c-Fos. It would be valuable to investigate and elucidate the further mechanisms of action that svAC3-33 functions through.

In conclusion, a novel splice variant of AC3-33 was successfully cloned, designated svAC3-33, which has a different structure and expression profile than AC3-33. Notably, svAC3-33 was predominantly expressed in the cytoplasm. Moreover, svAC3-33 inhibits MCF-7 cell cycle progression and the transcription of the AP-1 reporter gene by downregulating the expression of c-Jun, but not c-Fos, inhibiting MCF-7 cell proliferation.

\section{Acknowledgements}

Not applicable.

\section{Funding}

The present study was supported by the Natural Science Foundation of Hebei Province (grant nos. C2017209062 and H2018209140), and the National Natural Science Foundation of China (grant no. 81302323), and General Higher Education Young Talents Program of Hebei Province (grant no. BJ2014027).

\section{Availability of data and materials}

The datasets used and/or analyzed during the present study are available from the corresponding author on reasonable request.

\section{Authors' contributions}

LY, FH, YZ and XZ performed the RT-qPCR and western blot analysis. LM, TA and YC performed the in vitro experiment. LY, FH and XZ were major contributors in writing the manuscript. All authors read and approved the final manuscript. 


\section{Ethics approval and consent to participate}

Not applicable.

\section{Patient consent for publication}

Not applicable.

\section{Competing interests}

The authors declare that they have no competing interests.

\section{References}

1. Zhang X, Ma X, Xue Y, Meng L, He B, He S, Zhao J, Wang Y and Yang W: Prokaryotic expression and characterization of human AC3-33 protein. Front Biosci (Elite Ed) 2: 1134-1142, 2010.

2. Liu P, Deng WW, Gao P, Lu Y, Sun B, Li M, Zhao J, Shi TP and Zhang XJ: Molecular cloning and preliminary function study of a novel human gene AC3-33 related to suppress AP-1 activity. Yi Chuan 30: 575-582, 2008 (In Chinese).

3. Hao D, Gao P, Liu P, Zhao J, Wang Y, Yang W, Lu Y, Shi T and Zhang X: AC3-33, a novel secretory protein, inhibits Elk1 transcriptional activity via ERK pathway. Mol Biol Rep 38 : 1375-1382, 2011.

4. Wang ET, Sandberg R, Luo S, Khrebtukova I, Zhang L, Mayr C, Kingsmore SF, Schroth GP and Burge CB: Alternative isoform regulation in human tissue transcriptomes. Nature 456: 470-476, 2008.

5. Matlin AJ, Clark F and Smith CW: Understanding alternative splicing: Towards a cellular code. Nat Rev Mol Cell Bio 6 : 386-398, 2005

6. Wang P, Yu P, Gao P, Shi T and Ma D: Discovery of novel human transcript variants by analysis of intronic single-block EST with polyadenylation site. BMC Genomics 10: 518, 2009.

7. Valachovicova T, Slivova V, Bergman H, Shuherk J and Sliva D: Soy isoflavones suppress invasiveness of breast cancer cells by the inhibition of NF-kappaB/AP-1-dependent and -independent pathways. Int J Oncol 25: 1389-1395, 2004.

8. Zhou Y, Yau C, Gray JW, Chew K, Dairkee SH, Moore DH Eppenberger U, Eppenberger-Castori S and Benz CC: Enhanced NF kappaB and AP-1 transcriptional activity associated with antiestrogen resistant breast cancer. BMC Cancer 7: 59, 2007.

9. Liu Q, Song YJ, Meng LJ, Hu F, Gou LX, Jia CH, Tang HM, Wang WJ, Li M, Zhang XJ and Jia MC: Role of LM23 in cell proliferation and apoptosis and its expression during the testis development. Asian J Androl 15: 539-544, 2013.

10. Guo L, Chen C, Shi M, Wang F, Chen X, Diao D, Hu M, Yu M, Qian L and Guo N: Stat3-coordinated Lin-28-let-7-HMGA2 and miR-200-ZEB1 circuits initiate and maintain oncostatin M-driven epithelial-mesenchymal transition. Oncogene 32 : $5272-5282,2013$

11. Liu Y, Ludes-Meyers J, Zhang Y, Munoz-Medellin D, Kim HT, Lu C, Ge G, Schiff R, Hilsenbeck SG, Osborne CK and Brown PH: Inhibition of AP-1 transcription factor causes blockade of multiple signal transduction pathways and inhibits breast cancer growth. Oncogene 21: 7680-7689, 2002.

12. Huang J, Shi T, Ma T, Zhang Y, Ma X, Lu Y, Song Q, Liu W, Ma D and Qiu X: CCDC134, a novel secretory protein, inhibits activation of ERK and JNK, but not p38 MAPK. Cell Mol Life Sci 65: 338-349, 2008

13. Byun HJ, Hong IK, Kim E, Jin YJ, Jeoung DI, Hahn JH, Kim YM, Park SH and Lee H: A splice variant of CD99 increases motility and MMP-9 expression of human breast cancer cells through the AKT-, ERK- and JNK-dependent AP-1 activation signaling pathways. J Biol Chem 281: 34833-473, 2006.

14. Sunters A, Madureira PA, Pomeranz KM, Aubert M, Brosens JJ, Cook SJ, Burgering BM, Coombes RC and Lam EW: Paclitaxel-induced nuclear translocation of FOXO3a in breast cancer cells is mediated by c-Jun NH2-terminal kinase and Akt. Cancer Res 66: 212-220, 2006
15. Philips A, Chalbos D and Rochefort H: Estradiol increases and anti-estrogens antagonize the growth factor-induced activator protein-1 activity in MCF7 breast cancer cells without affecting c-fosand c-jun synthesis. J Biol Chem 268: 14103-14108, 1993.

16. Hu F, Meng X, Tong Q, Liang L, Xiang R, Zhu T and Yang S BMP-6 inhibits cell proliferation by targeting microRNA-192 in breast cancer. Biochim Biophys Acta 1832: 2379-2390, 2013.

17. Zang S, Chen F, Dai J, Guo D, Tse W, Qu X, Ma D and Ji C: RNAi-mediated knockdown of Notch-1 leads to cell growth inhibition and enhanced chemosensitivity in human breast cancer. Oncol Rep 23: 893-899, 2010.

18. Sun B, Nishihira J, Yoshiki T, Kondo M, Sato Y, Sasaki F and Todo S: Macrophage migration inhibitory factor promotes tumor invasion and metastasis via the Rho-dependent pathway. Clin Cancer Res 11: 1050-1058, 2005.

19. Das R, Mahabeleshwar GH and Kundu GC: Osteopontin induces AP-1-mediated secretion of urokinase-type plasminogen activator through c-Src-dependent epidermal growth factor receptor transactivation in breast cancer cells. J Biol Chem 279: 11051-11064, 2004

20. Wang P, Sun B, Hao D, Zhang X, Shi T and Ma D: Human TMEM174 that is highly expressed in kidney tissue activates AP-1 and promotes cell proliferation. Biochem Biophys Res Commun 394: 993-999, 2010.

21. Livak KJ and Schmittgen TD: Analysis of relative gene expression data using real-time quantitative PCR and the 2(-Delta Delta C(T)) method. Methods 25: 402-408, 2001.

22. Lewis BP, Green RE and Brenner SE: Evidence for the widespread coupling of alternative splicing and nonsense-mediated mRNA decay in humans. Proc Natl Acad Sci USA 100: 189-192, 2003

23. Modrek B and Lee C: A genomic view of alternative splicing. Nat Genet 30: 13-19, 2002.

24. Graveley BR: Alternative splicing: Increasing diversity in the proteomic world. Trends Genet 17: 100-117, 2001.

25. Brett D, Hanke J, Lehmann G, Haase S, Delbrück S, Krueger S, Reich J and Bork P: EST comparison indicates $38 \%$ of human mRNAs contain possible alternative splice forms. FEBS Lett 474: 83-86, 2000

26. Ladd AN and Cooper TA: Finding signals that regulate alternative splicing in the post-genomic era. Genome Biol 3: reviews0008, 2002.

27. Zheng ZM: Regulation of alternative RNA splicing by exon definition and exon sequences in viral and mammalian gene expression. J Biomed Sci 11: 278-294, 2004.

28. Unoki M, Shen JC, Zheng ZM and Harris CC: Novel splice variants of ING4 and their possible roles in the regulation of cell growth and motility. J Biol Chem 281: 34677-34686, 2006.

29. Liao XH,Lu DL, Wang N,Liu LY, Wang Y,Li YQ, Yan TB, Sun XG, $\mathrm{Hu} \mathrm{P}$ and Zhang TC: Estrogen receptor a mediates proliferation of breast cancer MCF-7 cells via a p21/PCNA/E2F1-dependent pathway. FEBS J 281: 927-942, 2014.

30. Al-Saran N, Subash-Babu P, Al-Nouri DM, Alfawaz HA and Alshatwi AA: Zinc enhances CDKN2A, pRb1 expression and regulates functional apoptosis via upregulation of p53 and p21 expression in human breast cancer MCF-7 cell. Environ Toxicol Pharmacol 47: 19-27, 2016.

31. Wang L, Wang G, Yang D, Guo X, Xu Y,Feng B and Kang J: Euphol arrests breast cancer cells at the G1 phase through the modulation of cyclin D1,p21 and p27 expression. Mol Med Rep 8: 1279-1285, 2013.

32. Lee MN, Jung EY, Kwun HJ, Jun HK, Yu DY, Choi YH and Jang KL: Hepatitis $C$ virus core protein represses the p21 promoter through inhibition of a TGF-beta pathway. J Gen Virol 83: 2145-2151, 2002

33. Stepniak E, Ricci R, Eferl R, Sumara G, Sumara I, Rath M, Hui L and Wagner EF: c-Jun/AP-1 controls liver regeneration by repressing p53/p21 and p38 MAPK activity. Genes Dev 20: 2306-2314, 2006

34. Gallo A, Cuozzo C, Esposito I, Maggiolini M, Bonofiglio D, Vivacqua A, Garramone M, Weiss C, Bohmann D and Musti AM: Menin uncouples Elk-1, JunD and c-Jun phosphorylation from MAP kinase activation. Oncogene 21: 6434-6445, 2002.

This work is licensed under a Creative Commons Attribution-NonCommercial-NoDerivatives 4.0 International (CC BY-NC-ND 4.0) License. 